\title{
The Sun and Big G Measurements
}

\author{
Jose L. Parra \\ Department of Physics, Florida International University, Miami, FL, USA \\ Email: JLparra@fiu.edu
}

How to cite this paper: Parra, J.L. (2018) The Sun and Big G Measurements. Journal of Applied Mathematics and Physics, 6, 2397-2401.

https://doi.org/10.4236/jamp.2018.611202

Received: October 30, 2018

Accepted: November 24, 2018

Published: November 27, 2018

Copyright (C) 2018 by author and Scientific Research Publishing Inc. This work is licensed under the Creative Commons Attribution International License (CC BY 4.0).

http://creativecommons.org/licenses/by/4.0/

\begin{abstract}
On August $29^{\text {th }}, 2018$, a scientific team reported a measure of the Universal Gravitational Constant $\mathrm{G}$ with the highest precision ever. The team completed three experimental campaigns in the same city over the course of a year. That work provided a complete data set useful analyzing the values of Big G change with the distance to the Sun, as is claimed by the author of this paper.
\end{abstract}

\section{Keywords \\ Gravitational Constant G, Rotation, Precession, Solar Cycles}

\section{Introduction}

This note is not exactly an independent paper. The original intention was to create a brief communication to show the correlation between the experimental data from two different experimental works. The author apologizes for lack of details on this communication about the Big $\mathrm{G}$ issue and recommends that interested readers check the reference for a complete overview on this point.

On August $29^{\text {th }}, 2018$, a scientific team reported a measure of the Universal Gravitational Constant, also known as Big G, with the lowest error ever. In this journal, Li et al. [1] announced two new values for Big G using two independent settings. The values for $\mathrm{G}$ were determined to be $6.674184(12) \times 10^{-11} \mathrm{~m}^{3} \cdot \mathrm{kg}^{-1} \cdot \mathrm{s}^{-2}$ using a technique called TOS and $6.674484(12) \times 10^{-11} \mathrm{~m}^{3} \cdot \mathrm{kg}^{-1} \cdot \mathrm{s}^{-2}$ using another technique called AAF. The physical nature of Big G is so goosy that after more than two years of science-art work, Li et al. exceed the precision reported in the work by Gundlach et al. [2] in the year 2000 [6.674215(92) $\left.\times 10^{-11} \mathrm{~m}^{3} \cdot \mathrm{kg}^{-1} \cdot \mathrm{s}^{-2}\right]$ by only ninety parts in a million. Many technological advances have occurred within the last 18 years, but the increment in precision between both studies is not well correlated with those advances. Another intriguing situation appears after comparing the numbers in red in the values reported by Li et al. The errors in both values cannot cover the $300^{\text {th }}$ of a million separations between them. It is 
not hard to imagine that this situation indicates that something is missing or hidden.

Schlamminger [3] published a comment in Nature on August $29^{\text {th }}, 2018$, calling attention to the fact that the TOS and AAF experiments were done by the same people, in the same place, and close in time. He thinks this could be a good combination to solve the $\mathrm{G}$ puzzle and proposes that $\mathrm{Li}$ et al. carry out a study explaining why they observe two non-overlapping values. He clarifies that the explanation can help to guide future research. Schlamminger moves as far as proposing to all the scholars to keep an open mind in regards to this problem. He wrote " $A$ second possibility is that some unknown physics could explain the scatter in the published values. Although this possibility is, of course, the more exciting, it is also the less likely. Nevertheless, it should not be dismissed lightly".

Moreover, I published papers in 2017 [4] and 2018 [5] covering the Big G issue both theoretically and experimentally. Therein, I mentioned that an unnoticed correlation exists between the best $\mathrm{G}$ values reported in the last 30 years and the distance to the Sun. Thirty years is a long period, which made it more difficult to draw a comparison between the studies carried out in so many labs throughout the world. Li et al's work enabled a facilitated analysis because their work and my work were produced during the same period.

I used a modified Cavendish' balance with two strings instead of the original single string balance. The length and separation of the strings act as a mechanical filter allowing only microscopic oscillations. Nothing is moved by human decision in this setting. This dynamic permits a precise reading of any variation in the separation between the masses on the balance and the masses fixed to the ground. Thus, 650 variations were collected, one per day, due to the apparent attraction between the masses. Unfortunately, it was not possible to calculate the value for Big $G$ with this setting because two new variables appeared, as shown in Equation (1) (For details, see Equation (3) in [5]). The effect of the angular variable was cancelled out in regular gravitational experiments by alternating the position of the attractor masses. The other effect created by the distance to the Sun cannot be neutralized completely because of non-linear effects. Li et al. have obtained 29 precise values for Big G; however, they failed to study the systematic variations of those values throughout the years. Every team has plenty of information on one type but lack information on the other. An intelligent combination of both types of experimental information can produce a clear and precise pattern for this gravitational problem.

$$
G_{\text {exp }}=G_{N E}+G_{(a, \alpha)}\left(\frac{a}{r}\right)^{3} \operatorname{Cos}\left(\theta_{\text {torus }}\right)
$$

\section{Li et al. [1] Chronological Data}

The team of Li et al. failed to report any possible variations of their data by taking into account other variables that might affect the results such as time or the distance from the Sun. Figure 1 included only the AAF data for reasons 


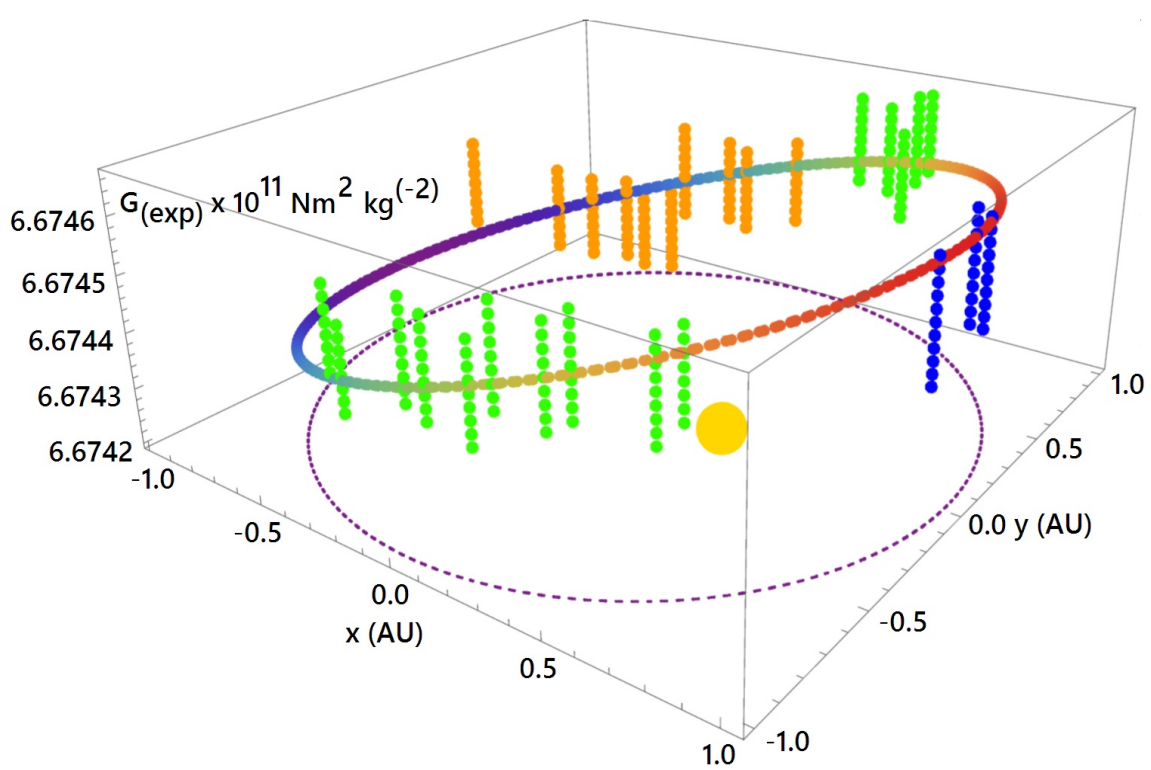

Figure 1. AAF error bars from three different campaigns with different color. The 2014-2015 campaign is shown in blue; the 2016 campaign is shown in orange, and the 2017-2018 campaign is shown in green.

explained later. A pattern is clear in Figure 1, despite only having $29 \mathrm{G}$ values. The figure included a dashed line representing the Earth's path, an offset yellow spot representing the Sun and a fitting curve. The red side of the fitting curve corresponds to the January-December period.

Figure 2 was created using the main values from AAF to introduce a fitting function. Figure 2 included only the $29 \mathrm{G}$ values obtained from the AAF experiments versus a unit-less parameter $x$ correlate with the distance to the Sun. The $x$ is the ratio between the instantaneous distance to the Sun and the average to that distance, also called the Astronomical Unit. Every value from AAF was determined after an average of three days. However, every value from TOS required an average of 132 days. That is an exclusionary condition in this analysis because such a large period hides progressive variations. This lengthy variation, which was not taken into account, can be used to explain why the final $G$ value determined using TOS is closer to the average G recommended by standard government departments.

Figure 1 also includes the regression function that corresponds to the theoretical model proposed by the author.

$$
G_{\text {exp. }}=\left\{(6.67218 \pm 0.00037)+\frac{0.00228 \pm 0.00036}{x^{3}}\right\} \times 10^{-11} \mathrm{~m}^{3} \mathrm{~kg}^{-1} \mathrm{~s}^{-2}
$$

In Equation (2), the value of $6.67218 \times 10^{-11} \mathrm{~m}^{3} \cdot \mathrm{kg}^{-1} \cdot \mathrm{s}^{-2}$ corresponds to the constant Big G. According to the author, the $0.00228(36) \times 10^{-11} \mathrm{~m}^{3} \cdot \mathrm{kg}^{-1} \cdot \mathrm{s}^{-2}$ value is due to the dragging effect arising from the nucleus of the Sun.

Now, it is possible to integrate the Big G data obtained by Li et al. with the variations recorded by the author. 


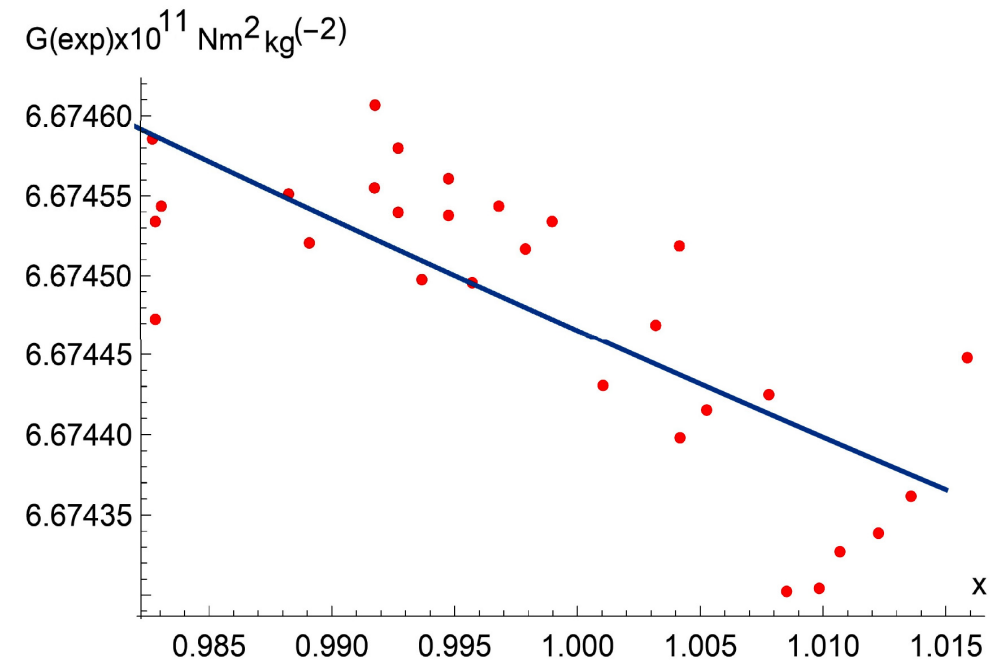

Figure 2. 29 values obtained by Li et al. using the AAF technique. The high vertical dispersion of the points should not impress negatively the reader because the precision on that axis is huge.

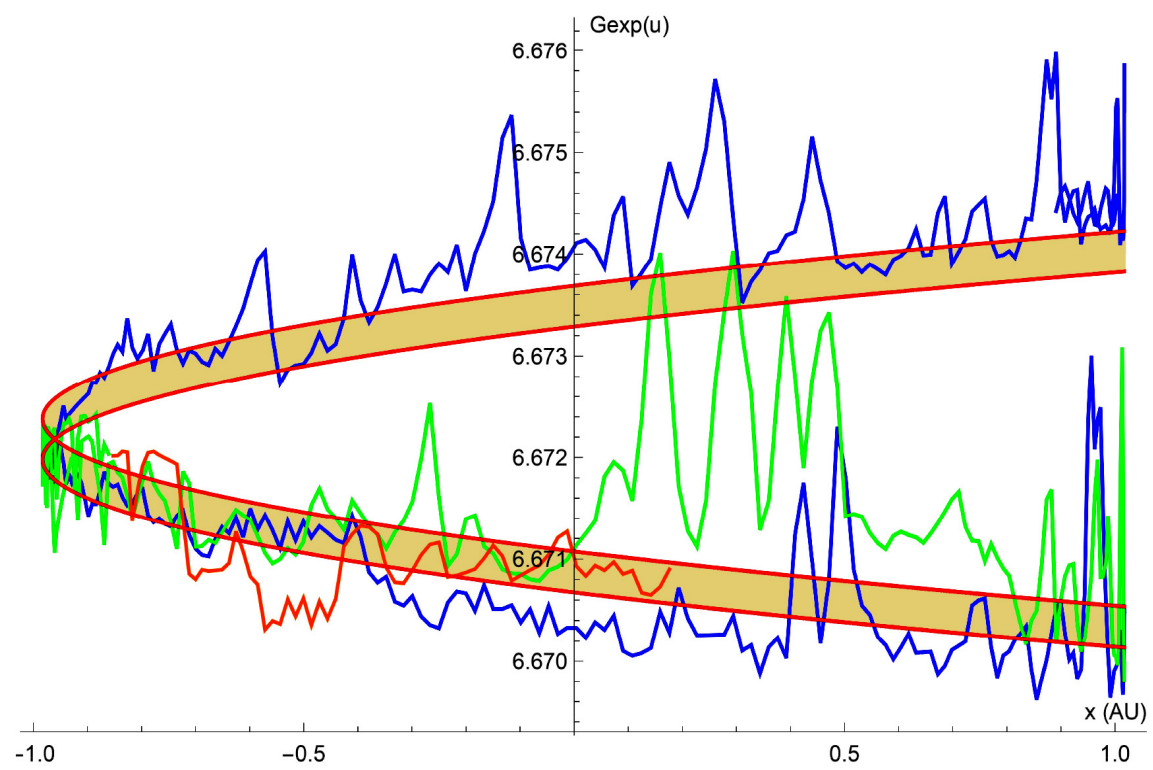

Figure 3. "Variations" of the attractive forces between the masses on a modified Cavendish' balance versus the distance to the Sun. The 24 Solar Cycle (SC) is shown in blue; the transition to the 25 SC (High oscillations) is shown in green, and the 25 SC is shown in orange.

\section{Mixing Big G Determinations and Its Variations}

The pattern in Figure 3 matches very well with Equation (1). The observed peaks are due to nutation' effects. Figure 3 includes a ribbon-like feature that is correlated to the fitting of Equation (2) but with new errors. The width of the ribbon is a visual representation of the new heightened precision. The ribbon was created using Equation (3) to contain the main sequence of the author's experimental data. 


$$
G_{\text {exp. }}=\left\{(6.67218 \pm 0.00020)+\frac{0.00228 \pm 0.00020}{x^{3}}\right\} \times 10^{-11} \mathrm{~m}^{3} \mathrm{~kg}^{-1} \mathrm{~s}^{-2}
$$

\section{Conclusion}

The average of the available experimental values of Big G can create confidence on its real value only to the third significant figure (6.67). Any researcher looking for more precise information about Big $G$ requires considering the experimental variation of its value with the distance to the Sun. This is experimentally justified by all the research conducted since Cavendish. Any art of science work in the modern time ought not aim or attempt to overcome this constraint. Many scholars may disagree with the assertion made in the author's initial hypothesis, but any argument can be brought in future work. In order to assert a different position on the hypothesis future work and research would have to be conducted; however, a six-significant figure on the value of Big G is due by now.

\section{Conflicts of Interest}

The author declares no conflicts of interest regarding the publication of this paper.

\section{References}

[1] Li, Q., et al. (2018) Measurements of the Gravitational Constant Using Two Independent Methods. Nature, 560, 582-588. https://doi.org/10.1038/s41586-018-0431-5

[2] Gundlach, J.H. and Merkowitz, S.M. (2000) Measurement of Newton's Constant Using a Torsion Balance with Angular Acceleration Feedback. Physical Review Letters, 85, 2869-2872. https://doi.org/10.1103/PhysRevLett.85.2869

[3] Schlamminger, S. (2018) Gravity Measured with Record Precision. Nature, 560, 562-563. https://doi.org/10.1038/d41586-018-06028-6

[4] Parra, J.L. (2017) The Implications of the Sun's Dragging Effect on Gravitational Experiments. International Journal of Astronomy and Astrophysics, 7, 174-184. https://doi.org/10.4236/ijaa.2017.73014

[5] Parra, J.L. (2018) Toward a Common Ground for Gravity and Optics. Journal of Applied Mathematics and Physics, 6, 1896-1906.

https://doi.org/10.4236/jamp.2018.69161 